\title{
Communications and Networking for Connected Vehicles 2020
}

\author{
Li Zhu $\mathbb{D}^{1},{ }^{1}$ Richard Yu $\mathbb{D}^{2},{ }^{2}$ Victor Leung $\mathbb{D}^{3},{ }^{3}$ Hongwei Wang, ${ }^{1}$ and Changqing Luo ${ }^{4}$ \\ ${ }^{1}$ State Key Lab. of Rail Traffic Control and Safety, Beijing Jiaotong University, Beijing, China \\ ${ }^{2}$ Department of Systems and Computer Eng., Carleton University, Ottawa, Canada \\ ${ }^{3}$ Department of Electrical and Computer Engineering University of British Columbia, Vancouver, Canada \\ ${ }^{4}$ Virginia Commonwealth University, Richmond, USA \\ Correspondence should be addressed to Li Zhu; zhulibjtu@gmail.com
}

Received 30 November 2021; Accepted 30 November 2021; Published 14 February 2022

Copyright (C) $2022 \mathrm{Li}$ Zhu et al. This is an open access article distributed under the Creative Commons Attribution License, which permits unrestricted use, distribution, and reproduction in any medium, provided the original work is properly cited.

With the new wave of urbanization, increasingly stringent emission standards, and intense pressure to improve the efficiency of private and public transportation, the development of more sustainable transportation systems becomes one of the fundamental social challenges of the next decade. Connected vehicles have been envisioned to provide enabling key technologies to enhance transportation efficiency, reduce incidents, improve safety, and mitigate traffic congestion impacts. The seamless integration and convergence of vehicular communication networks, information and transportation systems, and mobile devices and networks will face a number of technical, economic, and regulatory challenges. It is of paramount importance to (i) design vehicular communication systems that enable road users and other actors to exchange information in real-time with high reliability; (ii) enable pervasive sensing to monitor the status of vehicles and the surroundings; (iii) develop data analytics tools for processing large amounts of data generated by the connected vehicles; and (iv) develop middleware platforms for data management and sharing.

Within this context, we present a collection of highquality research papers presenting the latest developments, current research challenges, and future directions in the use of control, communication, and emerging technologies for safer and more efficient communication and networking of connected vehicles.

$Z$. Wang and his research fellows aim to achieve efficient bandwidth aggregation of heterogeneous wireless networks in vehicle-to-ground communication. A network coding scheme called Delay Determined Group Size (DDGS) is proposed for adaptively adjusting the coding group according to the heterogeneous wireless network state. The mathematical analysis and process design of the DDGS scheme are discussed in detail. A large number of simulations proved that the DDGS scheme is significantly superior to other coding group determination schemes in terms of decoding time cost and bandwidth aggregation efficiency. DDGS is very suitable for heterogeneous networks with low latency, high bandwidth links, high latency, and low bandwidth links in vehicle networks.

As one of the important parts of an intelligent transportation system (ITS), the V2V communication system allows vehicles to exchange information about the surrounding traffic situation to improve safety, reduce traffic congestion, and provide a comfortable driving experience. The channel characteristics are of vital importance in the research, design, and deployment of the $\mathrm{V} 2 \mathrm{~V}$ communication system. L. Xiong et al. in Beijing Jiaotong University investigate the vehicle-to-vehicle $(\mathrm{V} 2 \mathrm{~V})$ channel characteristics in peak hours at the $5.9 \mathrm{GHz}$ band in two typical urban road scenarios, the urban straight road and the intersection. The channel characteristics, such as path loss, root mean square (RMS) delay spread, and angular spread, are derived from the ray-tracing (RT) simulations. Due to the low height of antennas at both the transmitter $(\mathrm{Tx})$ and the receiver $(\mathrm{Rx})$, the line of sight (LOS) between the Tx and the Rx will often be obstructed by other vehicles. Based on the RT simulation results, the shadowing loss is modelled by the multimodal Gaussian distribution, and path loss models in both LOS and non-LOS (NLOS) conditions are obtained. And the RMS delay spread in two scenarios can be modelled by the Weibull distribution. In addition, the deployment of 
an antenna array is discussed based on the statistics distribution of the angular spread.

How to improve delay-sensitive traffic throughput is an open issue in vehicular communication networks, where a great number of vehicle to infrastructure (V2I) and vehicle to vehicle (V2V) links coexist. To address this issue, Y. Huang et al. in Chongqing University of Arts and Sciences propose a hybrid deep transfer learning scheme for allocating radio resources. Specifically, the traffic throughput maximization problem is first formulated by considering interchannel interference and statistical delay guarantee. The effective capacity theory is then applied to develop a power allocation scheme on each channel reused by a V2I and a V2V link. Thereafter, a deep transfer learning scheme is proposed to obtain the optimal channel assignment for each V2I and V2V link. Simulation results validate that the proposed scheme provides a close performance guarantee compared to a globally optimal scheme. Besides, the proposed scheme can guarantee lower delay violation probability than the schemes aiming to maximize the channel capacity.

Applying FD and $\mathrm{EH}$ techniques is inevitable for future wireless networks, especially for $\mathrm{V} 2 \mathrm{~V}$ communication systems, because of the big advantages of these techniques. Authors in Telecommunications University evaluate the ergodic capacity (EC) of full-duplex (FD) amplify-andforward (AF) and decode-and-forward (DF) relay system with energy harvesting (EH) for vehicle-to-vehicle (V2V) communications. Unlike previous works on FD-EH systems, the case that both relay and destination are mobile vehicles while the source is a static base station is considered. The exact closed-form expressions of ECs of both AF and DF protocols of the considered FD-EH-V2V relay system over cascade (double) Rayleigh fading are derived mathematically. Numerical results show that the ECs in the case of the $\mathrm{V} 2 \mathrm{~V}$ communication system are reduced compared to those in the case of stationary nodes. Also, with a specific value of residual self-interference (RSI), the ECs of the considered FD-EH-V2V relay system can be higher or lower than those of half-duplex- (HD-) EH-V2V system, depending on the average transmission power of the source. Furthermore, when the transmission power of the source and RSI are fixed, the ECs of the considered system can achieve peak values by using optimal $\mathrm{EH}$ time duration. On the other hand, the ECs of both AF and DF protocols reach the capacity floors in the high signal-to-noise ratio (SNR) regime due to the RSI impact. Also, the effect of RSI dominates the impact of cascade Rayleigh fading in the high SNR regime. Finally, the analysis approach is validated through Monte Carlo simulations.

Content-centric networks (CCNs) have become a promising technology for relieving the increasing wireless traffic demands. J. Ren et al. in Donghua University explore the scaling performance of mobile content-centric networks based on the nonuniform spatial distribution of nodes, where each node moves around its own home point and requests the desired content according to a Zipf distribution. The authors assume each mobile node is equipped with a finite local cache, which is applied to cache contents follow- ing a static cache allocation scheme. According to the nonuniform spatial distribution of cache-enabled nodes, the authors introduce two kinds of clustered models, i.e., the clustered grid model and the clustered random model. In each clustered model, we analyze throughput and delay performance when the number of nodes goes infinity by means of the proposed cell-partition scheduling scheme and the distributed multihop routing scheme. The authors show that the node mobility degree and the clustering behavior play the fundamental roles in the aforementioned asymptotic performance. Finally, the authors study the optimal cache allocation problem in the two kinds of clustered models. Their findings provide a guidance for developing the optimal caching scheme.

Communication-Based Train Control (CBTC) system is a critical signal system to ensure rail transit's safe operation. Compared with the train-ground CBTC system, the train control system based on train-to-train (T2T) communication has the advantages of fast response speed, simple structure, and low operating cost. As the core part of the train control system based on T2T communication, the reliability of the data communication system (DCS) is of great significance to ensure the train's safe and efficient operation. According to the T2T communication system requirements, H. Liang et al. in Beijing Jiaotong University adopt LongTerm Evolution-Unlicensed (LTE-U) technology to design the DCS structure and establishes the reliability model of the communication system based on Deterministic and Stochastic Petri Nets (DSPN). Based on testing the real line's communication performance parameters, the DSPN model is simulated and solved by $\Pi$-tool, and the reliability index of the system is obtained. The research results show that the LTE-U-based T2T communication system designed meets the train control system's needs for communication transmission. The proposed reliability evaluation method can complete the reliability modeling of train control DCS based on T2T communication. The research will provide a strong practical and theoretical basis for the design and optimization of train control DCS based on T2T communication.

Intelligent transportation system (ITS) is more and more crucial in the modern transportation field, such as the applications of autonomous vehicles, dynamic traffic light sequences, and automatic road enforcement. As the upcoming fifth-generation mobile network (5G) is entering the deployment phase, the idea of cellular vehicle-toeverything (C-V2X) is proposed. The same 5G networks, coming to mobile phones, will also allow vehicles to communicate wirelessly with each other. Hence, $3.5 \mathrm{GHz}$, as the main sub- $6 \mathrm{GHz}$ band licensed in $5 \mathrm{G}$, is focused. Y. Zeng et al. in Guangdong Communications \& Networks Institute conduct a comprehensive study on the channel characteristics for vehicle-to-infrastructure (V2I) link at $3.5 \mathrm{GHz}$ frequency band through channel measurements and raytracing (RT) simulations. Firstly, the channel parameters of the V2I link are characterized based on the measurements, including power delay profile (PDP), path loss, root-meansquare (RMS) delay spread, and coherence bandwidth. Then, the measurement-validated RT simulator is utilized 
to conduct the simulations in order to supplement other channel parameters, in terms of the Ricean $K$-factor, angular spreads, the cross-correlations of abovementioned parameters, and the autocorrelation of each parameter itself. This work is aimed at helping the researchers understand the channel characteristics of the V2I link at $3.5 \mathrm{GHz}$ and support the link-level and system level design for future vehicular communications of $5 \mathrm{G}$.

As one of the mainstream technologies of vehicle-toeverything (V2X) communication, Cellular-V2X (C-V2X) provides high-reliability and low-latency V2X communications. And with the development of mobile cellular systems, $\mathrm{C}-\mathrm{V} 2 \mathrm{X}$ is evolving from long-term evolution-V2X (LTEV2X) to new radio-V2X (NR-V2X). However, C-V2X test specification has not been completely set in the industry. In order to promote the formulation of relevant standards and accelerate the implementation of industrialization, Y. Fan et al. in Beijing Jiaotong University conduct the field test and analysis based on LTE-V2X in the industrial park scenario. Firstly, key technologies of LTE-V2X are introduced. Then, the specific methods and contents of this test are proposed, which consists of functional and network performance tests to comprehensively evaluate the communication property of LTE-V2X. Static and dynamic tests are required in both line-of-sight (LOS) and non-line-of-sight (NLOS) scenarios to evaluate network performance. Next, the test results verify that all functions are normal, and the performance evaluation indexes are appraised and analyzed. Finally, it summarizes the whole paper and puts forward the future work.

As an emerging type of Internet of Things (IoT), Internet of vehicles (IoV) denotes the vehicle network capable of supporting diverse types of intelligent services and has attracted great attention in the $5 \mathrm{G}$ era. K. Wang et al. in Xi'an University of Technology consider the multimedia content caching with multicast beamforming in IoV-based vehicular edge networks. First, the authors formulate a joint vehicle-to-vehicle- (V2V-) assisted clustering, caching, and multicasting optimization problem, to minimize the weighted sum of flow cost and power cost, subject to the quality-of-service (QoS) constraints for each multicast group. Then, with the two-timescale setup, the intractable and stochastic original problem is decoupled at separate timescales. More precisely, at the large timescale, the authors leverage the sample average approximation (SAA) technique to solve the joint $\mathrm{V} 2 \mathrm{~V}$-assisted clustering and caching problem and then demonstrate the equivalence of optimal solutions between the original problem and its relaxed linear programming (LP) counterpart; and at the small timescale, the authors leverage the successive convex approximation (SCA) method to solve the nonconvex multicast beamforming problem, whereby a series of convex subproblems can be acquired, with the convergence also assured. Finally, simulations are conducted with different system parameters to show the effectiveness of the proposed algorithm, revealing that the network performance can benefit from not only the power saving from wireless multicast beamforming in vehicular networks but also the content caching among vehicles.
The diversified service requirements in vehicular networks have stimulated the investigation to develop suitable technologies to satisfy the demands of vehicles. In this context, network slicing has been considered as one of the most promising architectural techniques to cater to the various strict service requirements. However, the unpredictability of the service traffic of each slice caused by the complex communication environments leads to a weak utilization of the allocated slicing resources. Thus, Y. Cui et al. in Chongqing University of Posts and Telecommunications use Long Short-Term Memory- (LSTM-) based resource allocation to reduce the total system delay. Specially, the authors first formulated the radio resource allocation problem as a convex optimization problem to minimize system delay. Secondly, to further reduce delay, the authors design a Convolutional LSTM- (ConvLSTM-) based traffic prediction to predict traffic of complex slice services in vehicular networks, which is used in the resource allocation processing. And three types of traffic are considered, that is, SMS, phone, and web traffic. Finally, based on the predicted results, i.e., the traffic of each slice and user load distribution, the authors exploit the primal-dual interior-point method to explore the optimal slice weight of resources. Numerical results show that the average error rates of predicted SMS, phone, and web traffic are $25.0 \%, 12.4 \%$, and $12.2 \%$, respectively, and the total delay is significantly reduced, which verifies the accuracy of the traffic prediction and the effectiveness of the proposed strategy.

With the rapid developments of wireless communication and increasing number of connected vehicles, Vehicular Ad Hoc Networks (VANETs) enable cyberinteractions in the physical transportation system. Future networks require real-time control capability to support delay-sensitive application such as connected autonomous vehicles. In recent years, fog computing becomes an emerging technology to deal with the insufficiency in traditional cloud computing. Z. Wang et al. propose a fog-based distributed network control design toward connected and automated vehicle application. The proposed architecture combines VANETs with the new fog paradigm to enhance the connectivity and collaboration among distributed vehicles. A case study of connected cruise control (CCC) is introduced to demonstrate the efficiency of the proposed architecture and control design. Finally, the authors discuss some future research directions and open issues to be addressed.

A vehicular cloud (VC) can reduce latency and improve resource utilization of the Internet of vehicles by effectively using the underutilized computing resources of nearby vehicles. Although the task offloading of the VC enhances road safety and traffic management on the Internet of vehicles and meets the low-latency requirements for driving safety services on the Internet of vehicles business, there are still some key challenges such as the resource allocation mechanism of differentiated services (DiffServ) and task offloading mechanism of improving user experience. To address these issues, Y. Kang et al. in Chongqing University of Posts and Telecommunications study the task offloading and resource allocation strategy of the VC system where tasks generated by vehicles can be offloaded and executed cooperatively by 
vehicles in VC. Specifically, the computing task is further divided into independent subtasks and executed in different vehicles in VC to maximize the offloading utility. Considering the mobility of vehicles, the deadline of tasks, and the limited computing resources, the authors propose the optimization problem of task offloading in the VC system in the cause of improved user experience. To characterize the difference in service requirements resulting from the diversity of tasks, a DiffServ model focusing on the pricing of a task is utilized. The initial pricing of a task is tailored by the characteristics of the task and the uniqueness of the network status. In this model, tasks are sorted and processed in order according to task pricing, so as to optimize resource allocation. Numerical results show that the proposed scheme can effectively increase the resource utilization and task completion ratio.

Mobile robot localization has attracted substantial consideration from the scientists during the last two decades. Mobile robot localization is the basics of successful navigation in a mobile network. Localization plays a key role to attain a high accuracy in mobile robot localization and robustness in vehicular localization. For this purpose, a mobile robot localization technique is evaluated to accomplish a high accuracy. This paper provides the performance evaluation of three localization techniques named Extended Kalman Filter (EKF), Unscented Kalman Filter (UKF), and Particle Filter (PF). I. Ullah et al. in Hohai University (HHU) propose three localization techniques. The performance of these three localization techniques is evaluated and analyzed while considering various aspects of localization. These aspects include localization coverage, time consumption, and velocity. The abovementioned localization techniques present a good accuracy and sound performance compared to other techniques.

In summary, it is essential that we continue to progress in the search for appropriate models that can adequately and faithfully improve the security and performance of Communications and Networking for Connected Vehicles. The progress reported in this special edition suggests that in the future, achieving these aims might be a distant prospect, but an attainable one.

\section{Conflicts of Interest}

The editors declare that they have no conflicts of interest regarding the publication of this special issue.

\section{Funding}

This article is supported by project $2021 \mathrm{CZ} 107$.

Li Zhu

Richard $Y u$

Victor Leung

Hongwei Wang

Changqing Luo 